Cultures \& Conflits

12 | hiver 1993

L'action collective : terrains d'analyse

\title{
Editorial : Identité et action collective
}

\section{La rédaction}

\section{(2) OpenEdition}

Journals

\section{Édition électronique}

URL : http://journals.openedition.org/conflits/435

DOI : $10.4000 /$ conflits.435

ISSN : $1777-5345$

Éditeur :

CCLS - Centre d'études sur les conflits lilberté et sécurité, L'Harmattan

Édition imprimée

Date de publication : 15 décembre 1993

ISSN : 1157-996X

Référence électronique

La rédaction, « Editorial : Identité et action collective », Cultures \& Conflits [En ligne], 12 | hiver 1993, mis en ligne le 14 mars 2006, consulté le 30 mars 2021. URL : http://journals.openedition.org/conflits/435 ; DOI : https://doi.org/10.4000/conflits.435

Ce document a été généré automatiquement le 30 mars 2021.

Creative Commons License 


\title{
Editorial : Identité et action collective
}

\author{
La rédaction
}

Ce numéro de Cultures \& Conflits, tout comme celui sur les conflits après la bipolarité, a été constitué à partir d'articles reçus par la rédaction indépendamment les uns des autres $^{1}$. On n'y cherchera donc pas une problématique construite comme dans les numéros à thèmes précédents. Néanmoins certaines grandes lignes se dégagent autour de la manière de penser l'action collective et traduisent une sensibilité accrue à "l'art de se regrouper" pour reprendre la formule de Guillaume Courty. Sans prétendre être autre chose que des pistes de recherche, ce numéro se propose, à partir de cas d'espèces, de renouveler la question des relations entre identité et action collective. Dipak Gupta à propos des Etats-Unis, Dominique Wisler de la Suisse, Gilles Bataillon du Nicaragua, Richard Banegas des transitions à l'Est, Guillaume Courty des barrages routiers, et dans une perspective plus philosophique Ayse Ceyhan à propos du communautarisme, se confrontent à cette question de l'identité et de l'action collective en proposant de nouvelles façons d'articuler cette relation.

L'une des principales interrogations auxquelles l'analyse de l'action collective est communément appelée à répondre est celle relative aux causes qui peuvent expliquer l'apparition, à tel ou tel moment, d'une mobilisation de la part de tel ou tel groupe d'individus. L'une des approches les plus classiques, celle vers laquelle on se dirige spontanément, consiste à partir des instants qui ont vu naître, vivre, et le cas échéant "mourir" une action commune, et à remonter en amont vers ce qui pourrait être susceptible de constituer une des raisons de l'action collective. On est ainsi amené à construire des chaînes de causalités plus ou moins longues, ou complexes. On cherche à établir une liste de conditions préexistantes sans lesquelles l'action collective ne saurait avoir lieu. Dès lors on a tendance aussi à hypostasier l'identité du groupe en amont, à le considérer comme donné dès le début de l'analyse. Il en résulte deux apories majeures: d'une part le repérage des conditions préalables est souvent inopérant, car celles-ci n'expliquent en rien les processus du passage à l'action collective puisque, dans de très nombreux cas, alors même que ces conditions semblent réunies, il ne se passe rien, d'autre part on pose un "gap" entre ces conditions 
préalables et le résultat, qui empêche de comprendre que les identités fluctuent au cours de la dynamique, qu'elles se font et se défont au cours de la relation. Michel Dobry a parfaitement montré les deux faiblesses de cette illusion étiologique ${ }^{2}$. Mais sortir des raisonnements causalistes ou finalistes n'est pas si simple qu'il y parait. Ceuxci viennent souvent hanter l'analyse qui disait les exclure. Charles Tilly dans "réclamer viva voce" expliquait à quel point il est difficile de se départir de cette vue fixiste et causaliste pour passer à une approche relationnelle et dynamique ${ }^{3}$. Un premier changement de perspective, qui n'est certes pas nouveau, mais qui souligne la faiblesse explicative des thèses sur les frustrations relatives, réside dans le renversement de la question de Ted Gurr dans plusieurs de ses ouvrages : pourquoi les hommes ou les minorités (ne) se révoltent-ils (pas plus souvent) ${ }^{4}$ ? C'est sous cet angle que Dipak Gupta s'interroge sur l'absence d'action collective des communautés noires américaines. Il montre incontestablement que la situation économique, sociale et politique de la majorité des membres des "communautés noires" n'entraîne pas de passage à l'acte, malgré les prédictions apocalyptiques de la commission Kerner - mise en place peu de temps après les émeutes urbaines de l'été 1967 afin d'en étudier les causes - prédictions qui auraient dû d'autant plus se réaliser que les conditions se sont depuis objectivement dégradées (comme le souligne la Commission Webster mise en place après les émeutes de Los Angeles de 1992). Sans considérer ces émeutes comme un épiphénomène, elles ne constituent donc pas pour Dipak Gupta la suite logique de la dégradation des conditions socio-politiques des communautés noires américaines et une vérification des thèses de Gurr, mais au contraire leur réfutation. Dipak Gupta s'engage alors dans une discussion sur les relations qui unissent l'action collective et l'identité des acteurs. Il propose une solution formaliste, tirée des modèles économétriques et de l'idée du partage du temps entre les moments consacrés à l'action individuelle et les moments consacrés à l'action collective. L'identité est alors formée pour lui de l'identité individuelle et de l'identité collective, identités qui peuvent être distinguées. Seulement en mettant en concurrence l'identité collective et l'identité individuelle, il maintient une différence qui écartèle sa problématique et on peut se demander s'il ne retombe pas dans le piège du "free rider". Par ailleurs il montre que l'identité du nous ne se structure pas indépendamment de celle du "eux" et qu'aucune identité collective ne peut être étudiée si l'on n'intègre pas la manière dont l'Etat, la police jouent sur la formation de cette identité. L'identité collective est alors toujours relationnelle, jamais donnée.

Dominique Wisler, en s'inspirant de manière critique des thèses de Hanspieter Kriesi sur les structures d'opportunité politique, n'est pas très éloigné de ce schéma d'analyse. Pour lui aussi les identités des mouvements se créent dans leur relation à l'Etat, et c'est en fonction de la réaction de celui-ci qu'ils pourront jouer des ressources habituelles de la mobilisation participation, ou qu'ils en seront exclus, et ce indépendamment $\mathrm{du}$ risque "objectif" qu'encourt l'Etat. Une optique par trop institutionnaliste et figée des structures d'opportunité politique, en terme d'ouverture ou de fermeture du système politique doit donc être remplacée par une approche en terme de sélectivité et de relation.

Gilles Bataillon dans son article sur les contras et les recontras nicaraguayens, va encore plus loin que les auteurs précédents dans la relativisation de la permanence des structures, en mettant l'accent sur la contextualisation. Pour lui, le "contexte" structure les identités collectives et individuelles surtout lorsque, dans l'exemple nicaraguayen, la polarisation ami/ennemi pousse chaque acteur à se définir autant 
contre l'autre que pour soi. Dès lors, la lecture des événements ne sauraient s'effectuer à la lumière d'actions extérieures, d'une culture ou d'un passé propre, sensés structurer en permanence les pratiques des acteurs engagés dans la crise nicaraguayenne. Les actions armées des contras, puis des recontras, et les formes qu'elles prennent, ne peuvent se comprendre qu'à la lumière de ce qui se passe, de ce qui se joue dans l'interaction. La structuration ami/ennemi n'est donc pas permanente, elle est contextuelle. Elle dépend des opportunités politiques des acteurs. Le rôle du Tiers, des tiers, un moment mis en parenthèse, redevient central pour comprendre tant les logiques de démocratisation que d'instrumentalisation d'une violence désenchantée, celle des recontras. Richard Banegas en s'appuyant fortement sur la thèse de Michel Dobry $^{5}$, reprend lui aussi cette approche en terme de contextualisation. Pour lui, il convient de s'intéresser à l'état conjoncturel des structures, aux propriétés contextuelles des configurations sociales, et à la manière dont celles-ci jouent sur la structuration des identités. Il rappelle l'aspect multidimensionnel de l'identité personnelle, et le fait que durant les périodes de "transitions démocratiques", périodes de crises, les mobilisations collectives sont caractérisées par une tendance à la simplification de cette identité, à une régression vers l'habitus, régression souvent utilisée par les entrepreneurs identitaires dans leurs stratégies de mobilisations antagonistes.

Guillaume Courty est sans doute, de tous les auteurs, le plus sensible à cette question de l'identité. Pour lui, toutes les approches précédentes présupposent, quelque part, malgré tout une identité afin de pouvoir penser l'action collective et font donc l'économie d'une réflexion sur "l'art de se regrouper", sur l'art d'être ensemble avant même ou pendant que l'on passe à l'action. Le biais objectiviste tendant à supposer des structures, des identités déjà là est alors dénoncé avec force. Le recours à l'histoire vise à casser cette image des structures structurantes. Ce qui importe ici, c'est de comprendre comment les identités collectives jouent de "modèles dramaturgiques" pour se constituer.

On doit sans doute à l'approche goffmanienne qui court comme un fil conducteur dans les différents travaux la forte critique des réifications objectivistes des identités et des structures. C'est la mise en oeuvre de ce paradigme qui a renouvelé l'approche en terme de mobilisation en mettant l'accent sur les stratégies et les dynamiques d'interaction. On a ajouté l'opportunité aux structures. Et c'est là un apport considérable. Mais jusqu'où peut-on aller dans cette voie? Dérouler un récit, comprendre les enjeux au niveau des interactions, les "mises en scènes de la vie quotidienne" suffit-il à rendre compte des processus de socialisation incorporés par les acteurs, des mécanismes relationnels qui s'imposent aux calculs des acteurs et des structures à long terme qui, comme la nature des trajectoires historiques de formation de l'Etat, influencent malgré tout l'ensemble de ces modalités de comportement? La tendance à croire que rien n'est stable, que tout se joue dans l'instant, que tout est fluide peut conduire à un biais subjectiviste qui oublie que les interactions (dont l'étude satisfait les empiristes, car on peut les observer, les filmer, les enregistrer, bref les toucher $\mathrm{du}_{\mathrm{doigt}}{ }^{6}$ ) cachent les structures qui s'y réalisent. Il faut sans doute rappeler que la vérité de l'interaction n'est jamais toute entière dans l'interaction telle qu'elle se livre à l'observation ${ }^{7}$. Le risque de l'objectivisme est grand, celui de l'éclatement du sujet ne l'est pas moins, sauf à renouer avec des croyances behaviouristes sur l'accumulation des connaissances à partir de micro-objets. Ne trouve-t-on pas alors chez Pierre Bourdieu, Norbert Elias, Charles Tilly, Doug Mac Adam, ou Sydney Tarrow 
un antidote à l'ethnométhodologie du courant goffmanien qui semble dominer actuellement nombre de travaux sur l'action collective en France? Des voies sont sans doute ouvertes pour penser l'incorporation des structures, pour analyser de manière dynamique leur émergence et les conditions de leur reproduction mais elles ressemblent au passage du Nord Ouest: Passage certes resserré, rare mais infiniment précieux car fractal ${ }^{8}$. A quand alors une approche fractale des identités et de l'action collective?

\section{NOTES}

1. Le nombre d'articles que nous avons reçus spontanément a dépassé de loin nos prévisions et nos possibilités de publication. Nous remercions tous ceux qui les ont envoyés et qui lisent la revue. Afin de préciser nos attentes envers nos auteurs potentiels signalons que les articles choisis par le comité de rédaction ont privilégié l'aspect théorique, en particulier lorsque celui-ci ressortait de l'analyse de cas d'espèces précis, et que nous avons écarté en revanche les purs "récits" ou "descriptions" ainsi que les articles trop "formalistes" et "généraux".

2. Cf. Sociologie des crises politiques, Presses de la FNSP, 1986, p. 48 et suiv.

3. Charles Tilly, "réclamer viva voce", Cultures \& Conflits, "Emeutes urbaines : le retour du politique", n5, printemps 1992.

4. Gurr (T. R.), Why men rebel ?, Princeton, N. J., Princeton university Press, 1970 ; Minorities at risk, USIP, Washington D.C., 1993.

5. op. cit.

6. Tout un courant de la sociologie des organisations demeure attaché à cette posture qui, sous prétexte d'éviter une généralisation abusive inhérente à ses yeux aux analyses en termes de structure, appréhende les actions des individus dans leur instantanéité, dans leur visibilité immédiate.

7. Pierre Bourdieu, "Espace social et pouvoir symbolique" in Choses dites, Minuit, 1987, p. 152.

8. Michel Serres, Le passage du Nord Ouest, Minuit, 1980.

INDEX

Mots-clés : identité, Mouvements sociaux 